\title{
Viscous perturbations of marginally stable Euler flow and finite-time Melnikov theory
}

\author{
Emmanuel Grenier \\ U.M.P.A. \\ Ecole Normale Supérieure de Lyon \\ 69364 Lyon Cedex 07, France \\ Frederic Rousset \\ C.N.R.S. \\ University of Nice - Sophia Antipolis \\ 06108 Nice Cedex 02, France
}

\author{
Christopher K.R.T. Jones \\ Department of Mathematics
}

University of North Carolina at Chapel Hill

Chapel Hill, NC 27599, USA

Björn Sandstede

Department of Mathematics

The Ohio State University

Columbus, OH 43202, USA

November 25, 2004

\begin{abstract}
The effect of small viscous dissipation on Lagrangian transport in two-dimensional vorticityconserving fluid flows motivates this work. If the inviscid equation admits a base flow in which different fluid regions are divided by separatrices, then transport between these regions is afforded by the splitting of separatrices caused by viscous dissipation. Finite-time Melnikov theory allows us to measure the splitting distance of separatrices provided the perturbed velocity field of the viscous fluid flow stays sufficiently close to vorticity-conserving base flow over sufficiently long time intervals. In this paper, we derive the necessary long-term estimates of solutions to Euler's equation and to the barotropic vorticity equation upon adding viscous perturbations and forcing. We discover that a certain stability condition on the unperturbed flow is sufficient to guarantee these long time estimates.
\end{abstract}




\section{Introduction}

Questions concerning the impact of adding viscosity and forcing to inviscid fluid flow arise naturally but are usually not simple to resolve due to the technical complications of the inviscid limit. One such question can be cast in terms of the Cauchy problem for two-dimensional flow: does the solution of the perturbed initial value problem, i.e. of the viscous forced equation, stay close for all, or very long, time to that of the unperturbed, i.e. inviscid and unforced, problem with the same initial value. Basic results (see, for instance [3, 10]) render results of the form: for a fixed time interval $[0, T]$ and under appropriate conditions on the unperturbed flow field, the viscous flow will tend, in an appropriate norm and at the rate of the perturbation parameter $\epsilon$, to the inviscid flow. The condition on the unperturbed flow is a regularity condition that relates to the absence of singularities, such as point vortices; results in the context of singular inviscid flows yield a weaker rate of convergence, see [19].

A natural question arises as to whether these results can be improved upon by imposing extra conditions on the unperturbed flow field. Of particular focus here is whether the time interval $T$ for convergence as $\epsilon \rightarrow 0$ can be related to $\epsilon$, i.e. whether $T=T_{\epsilon}$ and $T_{\epsilon} \rightarrow \infty$ as $\epsilon \rightarrow 0$. Our main result is that, with an appropriate stability condition on an unperturbed steady flow, a significant improvement can be made. Although the classical results are not stated in this way, it is not hard to obtain $T_{\epsilon} \sim|\ln \epsilon|$, and our main result improves upon this, under our stability conditions, to have $T_{\epsilon} \sim \epsilon^{-\kappa}$ for some $\kappa>0$.

Our specific motivation for considering this issue comes from a study of fluid transport in ocean flows. Models of basic ocean flows exhibiting features such as eddies or meandering jets are often given in terms of steady flow fields of inviscid Euler equations. Recent developments in dynamical systems ideas in the context of fluid flows have afforded an understanding of the fluid exchange between constituent parts of such a feature. It is well-known that even a simple two-dimensional fluid flow that is time-dependent can exhibit significant fluid exchange among the various flow regimes within features as well as between the feature and the ambient water. This phenomenon was cited by Samelson [14] as an explanation of the irregularity observed in float data in the North Atlantic. This work relies on the fact that the $\beta$-plane approximation to the Coriolis force added to the Euler equations can lead to a model with a jet that propagates in an easterly direction. Such a jet can be viewed as a simple model of such planetary jets as the Gulf Stream. This kind of base flow is, however, steady and hence exhibits no (chaotic) transport or mixing. Time-dependent perturbations will induce variations in the flow field that can "open" up avenues of fluid transport through breaking stable and unstable manifolds.

Time-dependence of the flow field is often put in artificially by making an appropriate parameter in the stream function depend on time. A much more satisfying approach is to take a simple model as a solution of the appropriate partial differential equation (PDE) and add physical effects to the model through embellishing the PDE accordingly. Through such an approach, we can then start to ask which physical effects might promote transport and in what ways. The underlying motivation for the work at hand is then to assess the influence of viscosity and forcing on the possibility and 
extent of fluid transport.

\subsection{Motivation: Transport in two-dimensional fluid flows}

Of interest then is the effect of viscous dissipation and forcing on two-dimensional vorticity conserving flows. The potential vorticity $q^{\varepsilon}(t, x, y)$ of the fluid satisfies the PDE

$$
\partial_{t} q^{\varepsilon}+\left\{\psi^{\varepsilon}, q^{\varepsilon}\right\}=\varepsilon\left[\Delta q^{\varepsilon}+f(t, x, y)\right]
$$

where

$$
\{f, g\}=\frac{\partial f}{\partial x} \frac{\partial g}{\partial y}-\frac{\partial g}{\partial y} \frac{\partial g}{\partial x}
$$

denotes the usual Poisson bracket. We think of the small parameter $0<\varepsilon \ll 1$ as a measure of the amount of viscous dissipation and forcing added to the vorticity-conserving equation. The stream function $\psi^{\varepsilon}$ and the potential vorticity $q^{\varepsilon}$ are related by

$$
q^{\varepsilon}=\Delta \psi^{\varepsilon}+\beta y
$$

where the term involving $\beta$ accounts for the Coriolis force. For the sake of simplicity, we will assume throughout this paper that (1.1) is posed on the torus $\mathbb{T}^{2}$ to avoid complications due to boundaries. Transport in the fluid is described by the ordinary differential equation (ODE)

$$
\frac{\mathrm{d}}{\mathrm{d} t}\left(\begin{array}{l}
x \\
y
\end{array}\right)=u^{\varepsilon}(t, x, y)
$$

that governs the dynamics of particles via their position $(x, y)(t)$, where the term $u^{\varepsilon}(t, x, y)$ on the right-hand side is the velocity field associated with the stream function $\psi^{\varepsilon}(t, x, y)$ via

$$
u^{\varepsilon}(t, x, y)=J \nabla \psi^{\varepsilon}(t, x, y), \quad J=\left(\begin{array}{rr}
0 & -1 \\
1 & 0
\end{array}\right) .
$$

We begin by discussing the unperturbed situation when $\varepsilon=0$. Since the PDE (1.1) then conserves vorticity, the ODE (1.3) behaves as if it were an autonomous integrable Hamiltonian system [3, 5].

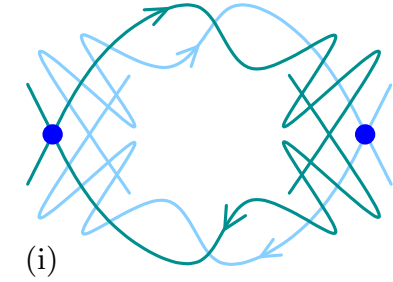

(i)

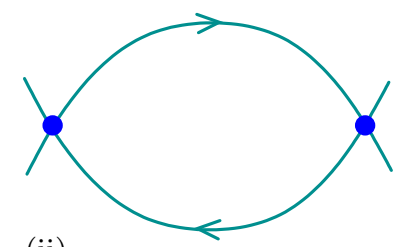

(ii)

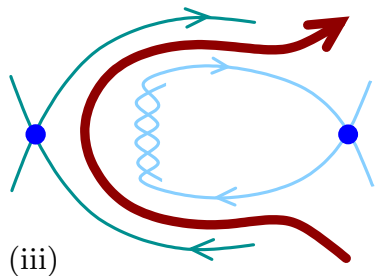

(iii)

Figure 1: The dynamics of (1.3) in the (x,y)-space is illustrated. The center plot (ii) shows trajectories when the vorticity-conserving base flow has a bounded recirculating region formed by two separatrices. Two different ways in which these separatrices may potentially break upon adding small viscous dissipation are illustrated in (i) and (iii): the left figure exhibits chaotic transport, while the right figure illustrates uni-directional fluid exchange. 
As a consequence, the unstable and stable manifolds of stagnation points will either coincide or not intersect at all. In particular, as shown in Figure 1(ii), heteroclinic loops may form that separate regions inside the fluid from the ambient fluid, thus precluding particle exchange between these regions and the ambient fluid. Upon allowing small viscous dissipation and forcing so that $0<\varepsilon \ll 1$, the heteroclinic loop may break so that the formerly separated regions inside and outside of the loop can exchange fluid particles. Figure 1(i) illustrates the situation where the stable and unstable manifolds intersect transversely for $\varepsilon>0$, thus leading to chaotic transport between adjacent fluid regions [12]. An alternative case, which leads to uni-directional transport across the formerly isolated spatial region, is shown in Figure 1(iii).

Melnikov theory can be used to predict which of these two cases occurs by allowing us to compute the splitting distance between stable and unstable manifolds via the Melnikov integral. To apply standard Melnikov theory, we need to know the perturbed velocity field $u^{\varepsilon}(t, x, y)$ explicitly to evaluate the Melnikov integral, and we have to show that $u^{\varepsilon}(t, x, y)$ is close, for all times, to the fluid flow $u^{0}(t, x, y)$ for $\varepsilon=0$. The second requirement is certainly unrealistic as the PDE (1.1) is parabolic for $\varepsilon>0$ so that solutions will, in general, not even exist in backward time. Computing the velocity field $u^{\varepsilon}(t, x, y)$ as a solution to (1.1) also appears to be difficult. We now briefly summarize the results in $[3,15]$ where we overcame these difficulties by developing a Melnikov theory for finite-time vector fields and showed that the Melnikov integral for (1.3) can be calculated without knowing the perturbed velocity field.

We assume that the stream function $\psi^{0}(t, x, y)$ is a travelling wave so that $\psi^{0}(t, x, y)=\Psi^{0}(x-c t, y)$ for some $c \in \mathbb{R}$ and some smooth function $\Psi^{0}(\xi, \eta)$. When rewritten using the variables $(\xi, \eta)=$ $(x-c t, y)$, equation (1.3) becomes autonomous. We assume that the transformed equation has, for $\varepsilon=0$, a trajectory $(\bar{\xi}, \bar{\eta})(t)=(\bar{x}(t)-c t, \bar{y}(t))$ that is homoclinic to the hyperbolic saddle $\left(\xi_{\infty}, \eta_{\infty}\right)$. Since we assumed that (1.1) is posed on $\mathbb{T}^{2}$, heteroclinic orbits can, in many cases, accommodated as well upon using periodicity in space, and we refer to $[3, \S 5]$ for a discussion of this point. The following hypothesis is crucial for our finite-time Melnikov theory.

Hypothesis 1 We assume that the difference $u^{\varepsilon}(t, x, y)-u^{0}(t, x, y)$ of the inviscid and viscid velocity fields of (1.1) is defined for every $\varepsilon \in\left[0, \varepsilon_{0}\right)$ and that, for appropriate numbers $\kappa>0$, $C>0$ and $\nu \in\left(\frac{1}{2}, 1\right]$, we have

$$
\left|u^{\varepsilon}(t, x, y)-u^{0}(t, x, y)\right|+\left|\nabla\left(u^{\varepsilon}(t, x, y)-u^{0}(t, x, y)\right)\right| \leq C \varepsilon^{\nu}
$$

uniformly in $0 \leq t \leq \varepsilon^{-\kappa}$ and $(x, y) \in \mathbb{T}^{2}$.

The following theorem gives the desired expression for the splitting distance that has been used in $[3,15]$ to predict the nature of transport for $0<\varepsilon \ll 1$.

Theorem 1 ([15]) Under the assumptions given above, the splitting distance of stable and unstable manifolds of (1.3) measured in the time slice $t=\tau$ is given by $\varepsilon M(\tau, \varepsilon)$ where

$$
M(\tau, \varepsilon)=\int_{-\infty}^{\infty}\left[\Delta q^{0}(t, \bar{x}(t), \bar{y}(t))-\Delta q^{0}\left(t, \bar{x}_{\infty}+c t, \bar{y}_{\infty}\right)\right] \mathrm{d} t
$$




$$
+\int_{-\infty}^{\infty}\left[f(t+\tau, \bar{x}(t), \bar{y}(t))-f\left(t+\tau, \bar{x}_{\infty}+c t, \bar{y}_{\infty}\right)\right] \mathrm{d} t+\mathrm{O}\left(\varepsilon^{2 \nu-1}\right)
$$

uniformly in $\tau \in\left[\frac{\nu}{\theta}|\ln \varepsilon|, \varepsilon^{-\kappa}-\frac{\nu}{\theta}|\ln \varepsilon|\right]$, where $\theta$ is the positive eigenvalue of $J \nabla \Psi^{0}\left(\xi_{\infty}, \eta_{\infty}\right)$.

Inspecting the Melnikov integral (1.5), we see that viscous dissipation alone leads to uni-directional transport as shown in Figure 1(iii), while genuinely time-dependent forcing may create chaotic transport as shown in Figure 1(i). We refer to [3, §8] for more details.

\subsection{Goal: Long-term estimates}

To apply Theorem 1, we need to establish the inequality (1.4) which requires an estimate of $\psi^{\varepsilon}-\psi^{0}$ that is valid for time intervals of length $\varepsilon^{-\kappa}$. From a PDE viewpoint, this raises the interesting question of long-time estimates for solutions of viscous and inviscid equations.

To explain this issue, we consider, as the simplest possible model, the two-dimensional Euler equation

$$
\partial_{t} u^{0}+\left(u^{0} \cdot \nabla\right) u^{0}+\nabla p^{0}=0, \quad \nabla \cdot u^{0}=0,
$$

and the Navier-Stokes equation

$$
\partial_{t} u^{\varepsilon}+\left(u^{\varepsilon} \cdot \nabla\right) u^{\varepsilon}+\nabla p^{\varepsilon}=\varepsilon\left[\Delta u^{\varepsilon}+f\right], \quad \nabla \cdot u^{\varepsilon}=0
$$

with small viscosity and a smooth bounded forcing term $f(t, x, y)$. In both equations, we consider $u^{\varepsilon}(t, x, y) \in \mathbb{R}^{2}$ with $(x, y)$ in the two-dimensional torus $\mathbb{T}^{2}$. As usual in the periodic setting, we shall consider flows $u^{0}$ and $u^{\varepsilon}$ that have zero mean on $\mathbb{T}^{2}$, hence we shall also assume that $\int_{\mathbb{T}^{2}} f=0$. Due to this last assumption, we can indeed express the velocity field $u^{\varepsilon}(t, x, y)$ via $u^{\varepsilon}=J \nabla \psi^{\varepsilon}$ in terms of a scalar stream function $\psi^{\varepsilon}(t, x, y)$. The vorticity $\omega^{\varepsilon}(t, x, y)$ defined by $\omega^{\varepsilon}=\Delta \psi^{\varepsilon}=\nabla \wedge u^{\varepsilon}$ will also play an important role in the analysis presented below, where $\nabla \wedge u:=\operatorname{det}[\nabla, u]$.

If $u_{\mathrm{s}}(x, y)$ is a stationary solution of (1.6), then the solution of (1.7) with initial condition $u_{\mathrm{s}}$ satisfies the estimate

$$
\left\|u^{\varepsilon}(t)-u_{\mathrm{s}}\right\|_{H^{1}\left(\mathbb{T}^{2}\right)} \leq \varepsilon C \mathrm{e}^{a t}
$$

for certain numbers $a>0$ and $C>0$, and we therefore have, for each $0<\gamma<1$, that

$$
\left\|u^{\varepsilon}-u_{\mathrm{s}}\right\|_{H^{1}\left(\mathbb{T}^{2}\right)} \leq \varepsilon^{\gamma} \quad \forall t \in\left[0, T_{\varepsilon}\right]
$$

where $T_{\varepsilon} \sim|\ln \varepsilon|$. The standard estimate (1.8) does not use any particular property of $u_{\mathrm{s}}$ and is true even if $u_{\mathrm{s}}$ is a bounded time-dependent solution of the Euler equation.

We expect that $T_{\varepsilon}$ will much larger when $u_{\mathrm{s}}$ is a linearly stable solution. A formal explanation goes as follows. If we set $u=u^{\varepsilon}-u_{\mathrm{s}}$ and neglect viscosity and nonlinear effects in the equation for $u$, we obtain a linear PDE of the form

$$
\partial_{t} u=\mathcal{A} u+\varepsilon F(t, x, y), \quad u(0, x, y)=0
$$


where $\mathcal{A}$ is the linearization of Euler's equation about $u_{\mathrm{s}}$, and $F=\nabla \wedge f+\Delta u_{\mathrm{s}}$. We consider (1.10) on the space $H_{\mathrm{df}}^{m}\left(\mathbb{T}^{2}\right)$ of functions in $H^{m}\left(\mathbb{T}^{2}\right)$ with $\nabla \cdot u=0$. Suppose now that $u_{\mathrm{s}}$ is a stable solution and that we can prove an estimate of the form

$$
\left\|\mathrm{e}^{\mathcal{A} t}\right\|_{L\left(H_{\mathrm{df}}^{1}\left(\mathbb{T}^{2}\right)\right)} \leq C \quad \forall t \geq 0
$$

for some $C>0$, then the mild solution

$$
u(t)=\varepsilon \int_{0}^{t} \mathrm{e}^{\mathcal{A}(t-s)} F(s) \mathrm{d} s
$$

of (1.10) satisfies the estimate $\|u(t)\|_{H^{1}\left(\mathbb{T}^{2}\right)} \leq \varepsilon C t$. Consequently, (1.9) is true for any $0<\gamma<1$ over time intervals of length $T_{\varepsilon} \sim \varepsilon^{\gamma-1}$. Furthermore, if $u_{\mathrm{s}}$ is weakly unstable so that

$$
\left\|\mathrm{e}^{\mathcal{A} t}\right\|_{L\left(H_{\mathrm{df}}^{1}\left(\mathbb{T}^{2}\right)\right)} \leq C t \quad \forall t \geq 0,
$$

then the mild solution (1.11) of (1.10) satisfies $\|u(t)\|_{H^{1}\left(\mathbb{T}^{2}\right)} \leq \varepsilon C t^{2}$, and (1.9) holds for $T_{\varepsilon} \sim \varepsilon^{(\gamma-1) / 2}$ which is good enough for (1.4) provided we choose $\gamma$ subject to $1 / 2<\gamma<1$.

The organization of this paper is as follows. In Section 2, we establish rigorously an estimate like (1.12) under appropriate assumptions on $u_{\mathrm{S}}(x, y)$ which are related to the Energy-Casimir approach to fluid stability due to Arnold [2]. In fact, we establish (1.12) in the equivalent $L^{2}$-norm of the vorticity. Next, we show that this estimate holds uniformly in the presence of vanishing viscosity and, building on this mild linear growth, we establish higher-order, weighted estimates for the full nonlinear equation (1.7) for $\varepsilon>0$ to prove that Hypothesis 1 is satisfied by this class of stationary flows $u_{\mathrm{s}}(x, y)$. We show in Sections 3 and 4 that these results apply also to the two-dimensional $\beta$-barotropic equation (1.1) and to modified Navier-Stokes equations for certain nonlinear viscosity stress tensors. In particular, in Section 3, we check Hypothesis 1 for the Rossby waves. Lastly, we summarize our results in Section 5.

\section{The Euler and Navier-Stokes equations}

Throughout this paper, we assume that $\int_{\mathbb{T}^{2}} f=0$ and consider only fluid flows with vanishing spatial average. In particular, we assume that $u_{\mathrm{s}}(x, y)$ is a smooth stationary solution of (1.6) with zero mean. We use the notation $\stackrel{H}{H}^{m}\left(\mathbb{T}^{2}\right):=\left\{\omega \in H^{m}\left(\mathbb{T}^{2}\right) ; \int_{\mathbb{T}^{2}} \omega=0\right\}$, and shall denote the $L^{2}$-norm and the $L^{2}$-scalar product on $\mathbb{T}^{2}$ by

$$
\|u\|^{2}=\langle u, u\rangle, \quad\langle u, v\rangle=\frac{1}{4 \pi^{2}} \int_{0}^{2 \pi} \int_{0}^{2 \pi} u(x, y) \cdot v(x, y) \mathrm{d} x \mathrm{~d} y .
$$

Lastly, various different constants that do not depend on $\varepsilon$ are denoted by $C$.

\subsection{Linear inviscid stability: The Euler equation}

We begin by recalling Arnold's approach [2] to nonlinear stability of stationary solutions of the Euler equation. The stream function $\psi_{\mathrm{s}}$ of any stationary solution of (1.6) is a function of the 
vorticity $\omega_{\mathrm{s}}=\Delta \psi_{\mathrm{s}}$ so that

$$
\psi_{\mathrm{s}}(x, y)=\Phi\left(\omega_{\mathrm{s}}(x, y)\right), \quad(x, y) \in \mathbb{T}^{2}
$$

for some, possibly multi-valued, function $\Phi$. We assume that $\Phi$ is single-valued and smooth. The classical approach [2, 9] for checking linear stability is to use the Hamiltonian structure of the Euler equation: consider the constrained Hamiltonian

$$
\mathcal{H}(u)=\int_{\mathbb{T}^{2}}\left(\frac{|u|^{2}}{2}+G(\omega)\right) \mathrm{d} x \mathrm{~d} y
$$

where $G$ is an anti-derivative of $\Phi=G^{\prime}$. The stationary solution $u_{\mathrm{s}}$ is a critical point of $\mathcal{H}$ so that $\mathrm{DH}\left(u_{\mathrm{s}}\right)=0$. Furthermore, the second variation

$$
\mathrm{D}^{2} \mathcal{H}\left(u_{\mathrm{s}}\right)[u, u]=\int_{\mathbb{T}^{2}}\left(|u|^{2}+G^{\prime \prime}\left(\omega_{\mathrm{s}}\right) \omega^{2}\right) \mathrm{d} x \mathrm{~d} y
$$

is conserved by the linearized Euler equation

$$
\partial_{t} u+\left(u_{\mathrm{s}} \cdot \nabla\right) u+(u \cdot \nabla) u_{\mathrm{s}}+\nabla p=0, \quad \nabla \cdot u=0 .
$$

Thus, we can conclude linear stability of $u_{\mathrm{s}}$ for perturbations with vorticity in $L^{2}$ provided the quadratic form $\mathrm{D}^{2} \mathcal{H}\left(u_{\mathrm{s}}\right)$ is a norm. According to [2], this is true

(i) if $G^{\prime \prime}\left(\omega_{\mathrm{s}}\right) \geq 0$, or

(ii) if there are positive numbers $\ell$ and $K$ such that $G^{\prime \prime}\left(\omega_{\mathrm{s}}\right) \leq-K$ and the Poincaré inequality $\|u\| \leq[K-\ell]\|\omega\|$ holds.

The first condition is related to the classical inflexion-point theorem of Rayleigh and Fjortoft [6, 13]. Although this method provides interesting results in various geometric settings (we refer to [2] or the book [11] for a survey), we see that the only stationary fluid flows on $\mathbb{T}^{2}$ that satisfy either one of the above conditions are the constant flows. Indeed, following Andrew's argument [1], we differentiate (2.1) with respect to $i=x, y$, multiply by $\partial_{i} \omega_{\mathrm{s}}$ and integrate by parts to get the identity

$$
\int_{\mathbb{T}^{2}}\left[G^{\prime \prime}\left(\omega_{\mathrm{s}}\right)\left(\partial_{i} \omega_{\mathrm{s}}\right)^{2}+\left|\partial_{i} u_{\mathrm{s}}\right|^{2}\right] \mathrm{d} x \mathrm{~d} y=0
$$

for $i=x, y$. It is now easy to see that $u_{\mathrm{s}}$ is constant whenever any one of the above two conditions is met. Thus, in our setting, we need to relax Condition (ii) to allow more interesting fluid flows. Associated with the quadratic form $\mathrm{D}^{2} \mathcal{H}\left(u_{\mathrm{s}}\right)$ is a symmetric linear operator $\mathcal{L}$ via

$$
\mathrm{D}^{2} \mathcal{H}\left(u_{\mathrm{s}}\right)[u, u]=\mathcal{N}(\omega)=\langle\omega, \mathcal{L} \omega\rangle
$$

which is, in fact, given by

$$
\mathcal{L} \omega=-G^{\prime \prime}\left(\omega_{\mathrm{s}}\right) \omega+\Delta^{-1} \omega,
$$

where $\Delta^{-1}$ is the operator defined by the Fourier series

$$
\Delta^{-1} \omega=-\sum_{(k, l) \neq(0,0)} \frac{\hat{\omega}_{k, l}}{k^{2}+l^{2}} \mathrm{e}^{\mathrm{i} k x+\mathrm{i} l y}
$$


on the Hilbert space $\stackrel{\circ}{L}^{2}\left(\mathbb{T}^{2}\right)$. Note that $\mathcal{L}$ is bounded and self-adjoint on $\stackrel{\circ}{L}^{2}\left(\mathbb{T}^{2}\right)$. We remark out that $\mathcal{L}$ is not the linearization about $u_{\mathrm{s}}$ of the Euler equation in vorticity form. However, there is a simple expression which relates the linearized equation (2.2) in vorticity form and the operator $\mathcal{L}$ : Due to (2.1), we have

$$
\partial_{t} \omega=-\left(u_{\mathrm{s}} \cdot \nabla \omega+u \cdot \nabla \omega_{\mathrm{s}}\right)=-\left(\partial_{y} \omega_{\mathrm{s}} \partial_{x}(\mathcal{L} \omega)-\partial_{x} \omega_{\mathrm{s}} \partial_{y}(\mathcal{L} \omega)\right)
$$

so that

$$
\partial_{t} \omega=\left\{\omega_{\mathrm{s}}, \mathcal{L} \omega\right\}
$$

where we used the Poisson bracket (1.2). In general, the spectra of $\mathcal{L}$, which is a bounded selfadjoint operator, and the linearized Euler equation in vorticity form given by (2.4) are not related. Nevertheless, the spectrum of $\mathcal{L}$ is useful in order to relax Condition (ii).

Since $L$ is self-adjoint, its spectrum is real. Furthermore, since $\mathcal{L}$ is a compact perturbation of the bounded multiplication operator $\omega \mapsto-G^{\prime \prime}\left(\omega_{\mathrm{s}}\right) \omega$, the essential spectrum $\Sigma_{\text {ess }}$ of $\mathcal{L}$ is given by $\left[-\sup G^{\prime \prime}\left(\omega_{\mathrm{s}}\right),-\inf G^{\prime \prime}\left(\omega_{\mathrm{s}}\right)\right]$. Consequently, if we assume that $G^{\prime \prime} \leq-K$, we obtain $\Sigma_{\text {ess }}(\mathcal{L}) \subset[K, \infty)$, and the spectrum of $\mathcal{L}$ in $(-\infty, K)$ consists only of isolated eigenvalues with finite multiplicity. Condition (ii) implies that the spectrum of $\mathcal{L}$ is, in fact, contained in $[\ell, \infty)$ where $\ell>0$. We shall relax Condition (ii) by allowing the situation where the smallest eigenvalue of $\mathcal{L}$ is equal to zero. More precisely, we assume that the following hypothesis is met.

Hypothesis 2 We assume that $G^{\prime \prime}\left(\omega_{\mathrm{s}}\right) \leq-K$ for some $K>0$ and that zero is a semi-simple eigenvalue of $\mathcal{L}$. Using the orthogonal decomposition

$$
\stackrel{\circ}{L}^{2}\left(\mathbb{T}^{2}\right)=\mathrm{N}(\mathcal{L}) \oplus \mathrm{R}(\mathcal{L}), \quad P: \stackrel{\circ}{L}^{2}\left(\mathbb{T}^{2}\right) \longrightarrow \mathrm{R}(\mathcal{L}),
$$

where $\mathrm{N}(\mathcal{L})=\operatorname{span}\left\{\omega_{1}, \ldots, \omega_{n}\right\}$, we assume that there is an $\ell>0$ such that

$$
\int_{\mathbb{T}^{2}} \omega \mathcal{L} \omega \geq \ell\|\omega\|^{2} \quad \forall \omega \in \mathrm{R}(\mathcal{L})
$$

Roughly speaking, Hypothesis 2 says that the functional $\mathcal{N}(\omega)=\langle\omega, \mathcal{L} \omega\rangle$ is semi-positive definite. The main result of this section states that Hypothesis 2 implies that $u_{\mathrm{s}}$ is at most algebraically unstable.

Proposition 1 Assume that Hypothesis 2 is met, then there exists a positive constant $C$ such that any solution $\omega(t)$ of (2.4) with $\omega(0, x, y)=\omega_{0}(x, y)$ satisfies the estimate

$$
\|\omega(t)\| \leq C(1+t)\left\|\omega_{0}\right\| \quad \forall t \geq 0 .
$$

Proof. We use the decomposition

$$
\omega(t, \cdot)=\sum_{j=1}^{n} a_{j}(t) \omega_{j}(\cdot)+\tilde{\omega}(t, \cdot)
$$


of $\omega$ given by Hypothesis 2, where $\tilde{\omega}=P \omega$ is the orthogonal projection of $\omega$ onto $R(\mathcal{L})$. Projecting (2.4) orthogonally onto $\mathrm{R}(\mathcal{L})$, we obtain

$$
\partial_{t} \tilde{\omega}=P\left\{\omega_{\mathrm{s}}, \mathcal{L} \tilde{\omega}\right\}
$$

and therefore

$$
\frac{1}{2} \frac{\mathrm{d}}{\mathrm{d} t} \mathcal{N}(\tilde{\omega})=\frac{1}{2} \frac{\mathrm{d}}{\mathrm{d} t}\langle\omega, \mathcal{L} \omega\rangle=\left\langle\mathcal{L} \tilde{\omega}, P\left\{\omega_{\mathrm{s}}, \mathcal{L} \tilde{\omega}\right\}\right\rangle=\left\langle\mathcal{L} \tilde{\omega},\left\{\omega_{\mathrm{s}}, \mathcal{L} \tilde{\omega}\right\}\right\rangle=0
$$

since $\{f,\{f, g\}\}=0$ for any two smooth functions $f$ and $g$. Thus, using (2.5), we see that there is a constant $C>0$ such that

$$
\ell\|P \omega(t)\|^{2} \leq \mathcal{N}(\tilde{\omega}(t))=\mathcal{N}\left(\tilde{\omega}_{0}\right) \leq C\left\|\omega_{0}\right\|^{2} .
$$

Next, projecting (2.4) orthogonally onto $\omega_{j}$, we obtain the equation

$$
\frac{\mathrm{d} a_{j}}{\mathrm{~d} t}(t)=\left\langle\left\{\omega_{\mathrm{s}}, \mathcal{L} P \omega\right\}, \omega_{j}\right\rangle
$$

for $a_{j}$ which, upon integrating by parts, gives

$$
\left|\frac{\mathrm{d} a_{j}}{\mathrm{~d} t}(t)\right| \leq C\|P \omega(t)\| \leq C\left\|\omega_{0}\right\| .
$$

Integrating this equation over time from zero to $t$, and using combining the result with (2.6) then gives the desired estimate.

Lastly, we give an example that satisfies Hypothesis 2.

Example 1 Consider the fluid flow defined by the stream function

$$
\psi_{\mathrm{s}}(x, y)=A \sin (x+a)+B \sin (y+b)
$$

on $\mathbb{T}^{2}$. We have $\Phi(X)=-X$ and therefore $\mathcal{L}=\mathbb{1}+\Delta^{-1}$. The spectrum of $\mathcal{L}$ consists entirely of eigenvalues given by

$$
\lambda_{k l}=1-\frac{1}{k^{2}+l^{2}}, \quad(k, l) \in \mathbb{Z}^{2} \backslash\{(0,0)\},
$$

and the null space of $\mathcal{L}$ is spanned by $\{\cos x, \sin x, \cos y, \sin y\}$. In particular, Hypothesis 2 is true thanks to Fourier-series expansions.

Note that the fluid flows in Example 1 exhibit closed stream lines, see Figure 2, and hence are interesting for oceanographic problems. The classical sinusoidal shear flow given by $\omega_{\mathrm{s}}(x, y)=\sin (y)$ is a special case of Example 1.

We point out that the fact that we can apply Proposition 1 to the family (2.7) of flows is compatible with the result in [16] which says that the essential spectrum of the linearized Euler operator in vorticity form lies on the imaginary axis when posed on $\stackrel{\circ}{L}^{2}$. In addition to excluding unstable point spectrum, Proposition 1 provides an estimate for the algebraic growth of solutions which does not 


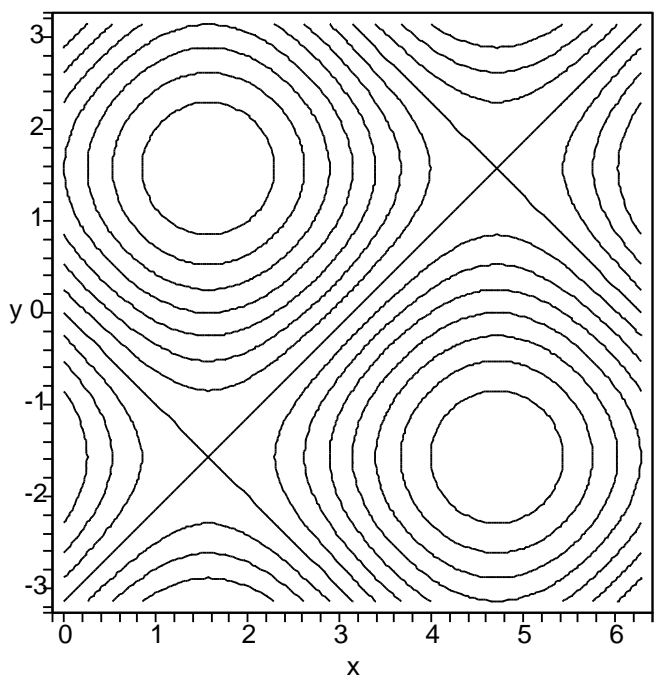

Figure 2: The level sets (streamlines) of the stream function $\psi_{\mathbf{s}}(x, y)=\sin x+\sin y$ are plotted.

follow directly from the absence of spectrum in the open right half-plane. We should also mention that it has been proved in $[16,20]$ that the essential spectrum is unstable on $\stackrel{\circ}{H}^{m}$ for each integer $m \neq 0$ in the vorticity formulation, while it is unstable on $H_{\mathrm{df}}^{m}$ for each integer $m \neq 1$ in the velocity formulation.

Lastly, we remark that the domain size is crucial for Hypothesis 2 to be true. For example, for the sinusoidal shear flow $\omega_{\mathrm{s}}(x, y)=\sin (k y)$ with $k>1$ on $\mathbb{T}^{2}$, Hypothesis 2 is not true anymore. In line with this, it has been shown in [8, Proposition 5.1] that the associated operator $\mathcal{A}$ has an eigenvalue with positive real part associated with a smooth eigenfunction, and $\mathrm{e}^{\mathcal{A} t}$ therefore grows exponentially on $H_{\mathrm{df}}^{m}\left(\mathbb{T}^{2}\right)$ for each $m \geq 0$.

\subsection{Viscous perturbations: The Navier-Stokes equations}

In this section, we investigate the effects of viscous perturbations on mildly unstable flows. We consider the solution $u^{\varepsilon}(t, x, y)$ of (1.7) with initial condition $u^{\varepsilon}(0, x, y)=u_{\mathrm{s}}(x, y)$ where $u_{\mathrm{s}}$ is again a smooth stationary solution of the Euler equation (1.6) with $\int_{\mathbb{T}^{2}} u_{\mathrm{s}}=0$.

Setting $u:=u^{\varepsilon}-u_{\mathrm{s}}$ and $\omega:=\nabla \wedge u$, we see that $\omega$ satisfies the equation

$$
\partial_{t} \omega+u_{\mathrm{s}} \cdot \nabla \omega+u \cdot \nabla \omega_{\mathrm{s}}=\varepsilon[\Delta \omega+F]-u \cdot \nabla \omega, \quad u=J \nabla \Delta^{-1} \omega,
$$

where $F=\nabla \wedge f+\Delta \omega_{\mathrm{s}}$, which can also be written as

$$
\partial_{t} \omega=\left\{\omega_{\mathrm{s}}, \mathcal{L} \omega\right\}+\varepsilon[\Delta \omega+F]-(u \cdot \nabla) \omega .
$$

The initial condition for $\omega$ is

$$
\omega(0, x, y)=0
$$


Lastly, using multi-indices $\alpha \in \mathbb{N}^{2}$ for derivatives with respect to $(x, y)$, we define the weighted norm

$$
E_{m}(t)=\sum_{0 \leq|\alpha| \leq m} \eta^{|\alpha|} \varepsilon^{|\alpha|}\left\|\partial^{\alpha} \omega(t)\right\|^{2}
$$

for an appropriate, sufficiently small constant $\eta>0$ that will be chosen later independently of $\varepsilon$.

Proposition 2 If Hypothesis 2 is met, then the solution $\omega(t)$ of (2.9)-(2.10) satisfies, for each fixed $\gamma \in\left(\frac{1}{2}, 1\right)$, the estimate

$$
E_{m}(t) \leq \varepsilon^{2 \gamma} \quad \forall t \in\left[0, T_{\varepsilon}\right]
$$

for $\varepsilon \in(0,1]$, where $T_{\varepsilon} \geq C \varepsilon^{\frac{\gamma-1}{3}}$.

Proof. Equation (2.9) has global solutions, hence we can define $T_{\varepsilon}$ to be the maximal time for which

$$
E_{m}(t)+\varepsilon \int_{0}^{t} D_{m}(s) \mathrm{d} s \leq \varepsilon^{2 \gamma} \quad \forall t \in\left[0, T_{\varepsilon}\right)
$$

where

$$
D_{m}(t)=\sum_{0 \leq|\alpha| \leq m} \eta^{|\alpha|} \varepsilon^{|\alpha|}\left\|\nabla P \partial^{\alpha} \omega(t)\right\|^{2} .
$$

We need to find a lower bound for $T_{\varepsilon}$. To get this bound, we shall prove that, assuming that (2.11) is true for some $\gamma \in(1 / 2,1)$, we actually have

$$
E_{m}(t)+\varepsilon \int_{0}^{t} D_{m}(s) \mathrm{d} s \leq C \varepsilon^{\frac{3 \gamma+1}{2}} T_{\varepsilon}^{3 / 2} \quad \forall t \in\left[0, T_{\varepsilon}\right)
$$

Before we show that (2.11) implies (2.12), let us note that (2.11)-(2.12) together imply that

$$
T_{\varepsilon} \geq C \varepsilon^{\frac{\gamma-1}{3}}
$$

which is the statement we wish to prove.

We begin with estimating $E_{0}(t)=\|\omega(t)\|^{2}$. To estimate $\|\omega\|$, we write

$$
\omega(t, x, y)=\sum_{j=1}^{n} a_{j}(t) \omega_{j}(x, y)+\tilde{\omega}(t, x, y),
$$

where $\tilde{\omega}=P \omega$. Projecting (2.9) onto the null space and the range of $P$, we obtain

$$
\frac{\mathrm{d} a_{j}}{\mathrm{~d} t}(t)=\varepsilon\left\langle\Delta \omega, \omega_{j}\right\rangle+\varepsilon\left\langle F, \omega_{j}\right\rangle+\left\langle\left\{\omega_{\mathrm{s}}, \mathcal{L} P \omega\right\}, \omega_{j}\right\rangle-\left\langle u \cdot \nabla \omega, \omega_{j}\right\rangle
$$

and

$$
\partial_{t} \tilde{\omega}+P\left\{\omega_{s}, \mathcal{L} \tilde{\omega}\right\}=P[\varepsilon(\Delta \omega+F)-u \cdot \nabla \omega] .
$$

First, we use (2.13) to estimate $a_{j}$. Integrating by parts, we get

$$
\left|\left\langle\left\{\omega_{\mathrm{s}}, \mathcal{L} P \omega\right\}, \omega_{j}\right\rangle\right| \leq C\|P \omega\| .
$$


Therefore, using that $\nabla \cdot u=0$ and that

$$
\|u\| \leq\left\|J \nabla \Delta^{-1} \omega\right\| \leq C\|\omega\|
$$

we obtain

$$
\begin{aligned}
\frac{1}{2} \frac{\mathrm{d}}{\mathrm{d} t}\left|a_{j}(t)\right|^{2} & =\varepsilon\left\langle\Delta \omega, \omega_{j}\right\rangle a_{j}+\varepsilon\left\langle F, \omega_{j}\right\rangle a_{j}+\left\langle\left\{\omega_{\mathrm{s}}, \mathcal{L} P \omega\right\}, \omega_{j}\right\rangle a_{j}-\left\langle u \cdot \nabla \omega, \omega_{j}\right\rangle a_{j} \\
& =\varepsilon\left\langle\omega, \Delta \omega_{j}\right\rangle a_{j}+\varepsilon\left\langle F, \omega_{j}\right\rangle a_{j}+\left\langle\left\{\omega_{\mathrm{s}}, \mathcal{L} P \omega\right\}, \omega_{j}\right\rangle a_{j}+\left\langle\omega, u \cdot \nabla \omega_{j}\right\rangle a_{j} \\
& \leq C\left[\|P \omega\|\|\omega\|+\varepsilon\|\omega\|+\|\omega\|^{3}\right] .
\end{aligned}
$$

As in the previous section, we use the bilinear form $\mathcal{N}(\tilde{\omega})$ to estimate $\tilde{\omega}(t)=P \omega(t)$. We obtain

$$
\frac{1}{2} \frac{\mathrm{d}}{\mathrm{d} t} \mathcal{N}(\tilde{\omega})=\frac{1}{2} \frac{\mathrm{d}}{\mathrm{d} t}\langle\mathcal{L} \tilde{\omega}, \tilde{\omega}\rangle=\left\langle\mathcal{L} \tilde{\omega}, \partial_{t} \tilde{\omega}\right\rangle=\langle P[\varepsilon \Delta \omega+\varepsilon F-u \cdot \nabla \omega], \mathcal{L} \tilde{\omega}\rangle
$$

since

$$
\left\langle P\left\{\omega_{\mathrm{s}}, \mathcal{L} \tilde{\omega}\right\}, \mathcal{L} \tilde{\omega}\right\rangle=\left\langle\left\{\omega_{\mathrm{s}}, \mathcal{L} \tilde{\omega}\right\}, \mathcal{L} \tilde{\omega}\right\rangle=0
$$

as $P$ is the orthogonal projection onto $\mathrm{R}(\mathcal{L})$. We now estimate the terms on the right-hand side of (2.15). First, note that

$$
\varepsilon\langle P F, \mathcal{L} \tilde{\omega}\rangle \leq C \varepsilon\|\omega\|
$$

To estimate the viscous term, we write

$$
\begin{aligned}
\varepsilon\langle P \Delta \omega, \mathcal{L} \tilde{\omega}\rangle & \leq \varepsilon\langle\Delta \omega, \mathcal{L} \omega\rangle=-\varepsilon\langle\nabla \omega, \mathcal{L} \nabla \omega\rangle+\varepsilon\left\langle\nabla \omega, \omega \nabla G^{\prime \prime}\left(\omega_{\mathrm{s}}\right)\right\rangle \\
& \leq-\varepsilon \ell\|P \nabla \omega\|^{2}+\varepsilon C\|\nabla \omega\|\|\omega\| \\
& \leq-\varepsilon \ell\|P \nabla \omega\|^{2}+\varepsilon C\|P \nabla \omega\|\|\omega\|+\varepsilon C\|(\mathbb{1}-P) \nabla \omega\|\|\omega\| .
\end{aligned}
$$

Since $[\mathbb{1}-P]$ projects onto the finite-dimensional null space $\mathrm{N}(\mathcal{L})$, we obtain $\|(\mathbb{1}-P) \nabla \omega\| \leq C\|\omega\|$, and using Young's inequality finally gives the estimate

$$
\varepsilon\langle P \Delta \omega, \mathcal{L} \tilde{\omega}\rangle \leq-\frac{\varepsilon \ell}{2}\|P \nabla \omega\|^{2}+C \varepsilon\|\omega\|^{2} .
$$

Thus, it remains to estimate the nonlinear term in (2.15). Integrating by parts and using that $\nabla \cdot u=0$, we obtain

$$
\begin{aligned}
-\langle P(u \cdot \nabla \omega), \mathcal{L} \tilde{\omega}\rangle & =\langle\omega, u \cdot \nabla(\mathcal{L} \omega)\rangle \\
& =\left\langle\omega, u \cdot\left[-\omega \nabla\left(F^{\prime \prime}\left(\omega_{\mathrm{s}}\right)\right)-F^{\prime \prime}\left(\omega_{\mathrm{s}}\right) \nabla \omega+\nabla \Delta^{-1} \omega\right]\right\rangle \\
& =-\frac{1}{2}\left\langle|\omega|^{2} u, \nabla F^{\prime \prime}\left(\omega_{\mathrm{s}}\right)\right\rangle+\left\langle\omega, u \cdot \nabla \Delta^{-1} \omega\right\rangle
\end{aligned}
$$

and therefore

$$
|\langle P(u \cdot \nabla \omega), \mathcal{L} \tilde{\omega}\rangle| \leq C\|u(t)\|_{L^{\infty}}\|\omega\|^{2} .
$$

We now establish a Gagliardo-Nirenberg inequality to estimate the $L^{\infty}$-norm of $u$. Using the Fourier series of $u$, and applying Hölder's inequality twice (first with $p=q=2$ and afterwards 
with $1 / p=\theta$ and $1 / q=1-\theta)$, we get

$$
\begin{aligned}
\|u(t)\|_{L^{\infty}} & \leq \sum_{(k, l) \neq(0,0)}\left|\hat{u}_{k, l}\right| \leq \sum_{(k, l) \neq(0,0)}\left[\left(k^{2}+l^{2}\right)\left|\hat{u}_{k, l}\right|\right]^{\theta}\left|\hat{u}_{k, l}\right|^{1-\theta}\left(\frac{1}{k^{2}+l^{2}}\right)^{\theta} \\
& \leq\|\Delta u\|^{\theta}\|u\|^{1-\theta}\left(\sum_{(k, l) \neq(0,0)}\left(\frac{1}{k^{2}+l^{2}}\right)^{2 \theta}\right)^{\frac{1}{2}} \\
& \leq C\|\Delta u\|^{\theta}\|u\|^{1-\theta} \leq C\|\nabla \omega\|^{\theta}\|\omega\|^{1-\theta}
\end{aligned}
$$

for any $1 / 2<\theta \leq 1$. Substituting this estimate in (2.19), we conclude that

$$
|\langle P(u \cdot \nabla \omega), \mathcal{L} \tilde{\omega}\rangle| \leq C\|\omega\|^{3-\theta}\|\nabla \omega\|^{\theta} .
$$

Setting $\theta=3 / 4$ and using again Young's inequality, we obtain

$$
\begin{aligned}
|\langle P(u \cdot \nabla \omega), \mathcal{L} \tilde{\omega}\rangle| & \leq C[\sqrt{\varepsilon}\|\nabla \omega\|]^{3 / 4}\left[\frac{\|\omega\|^{9 / 4}}{\varepsilon^{3 / 8}}\right] \leq \delta \varepsilon\|\nabla \omega\|^{2}+C(\delta) \frac{\|\omega\|^{18 / 5}}{\varepsilon^{3 / 5}} \\
& \leq \delta \varepsilon\|P \nabla \omega\|^{2}+C(\delta)\left[\varepsilon\|\omega\|^{2}+\frac{\|\omega\|^{18 / 5}}{\varepsilon^{3 / 5}}\right] .
\end{aligned}
$$

Substituting the estimates (2.17), (2.18) and (2.21) into (2.15), we get

$$
\frac{1}{2} \frac{\mathrm{d}}{\mathrm{d} t} \mathcal{N}(\tilde{\omega}) \leq \varepsilon\left[\delta-\frac{\ell}{2}\right]\|P \nabla \omega\|^{2}+C(\delta)\left[\varepsilon\|\omega\|+\varepsilon\|\omega\|^{2}+\frac{\|\omega\|^{18 / 5}}{\varepsilon^{3 / 5}}\right] .
$$

Choosing $\delta=\ell / 4$ and using (2.5), (2.10) and (2.11), we obtain

$$
\|P \omega(t)\|^{2}+\varepsilon \int_{0}^{t}\|P \nabla \omega(s)\|^{2} \mathrm{~d} s \leq C \int_{0}^{t}\left[\varepsilon\|\omega\|+\varepsilon\|\omega\|^{2}+\frac{1}{\varepsilon^{3 / 5}}\|\omega\|^{18 / 5}\right] \mathrm{d} s \leq C T_{\varepsilon} \varepsilon^{1+\gamma}
$$

uniformly in $t \in\left[0, T_{\varepsilon}\right)$. In particular, we have

$$
\|P \omega(t)\| \leq C T_{\varepsilon}^{1 / 2} \varepsilon^{\frac{1+\gamma}{2}},
$$

so that (2.14) becomes

$$
\left|a_{j}(t)\right|^{2} \leq C T_{\varepsilon}^{3 / 2} \varepsilon^{\frac{1+3 \gamma}{2}} .
$$

Combining (2.22) and (2.23), we obtain the desired estimate (2.12) for $m=0$.

The estimates (2.12) for $m \geq 1$ can now be obtained by classical energy methods. For instance, to estimate $\eta \varepsilon\left\|\partial_{x} \omega\right\|^{2}$, we take the $x$-derivative of (2.9), multiply by $\partial_{x} \omega$ and integrate over $\mathbb{T}^{2}$ which gives an expression for $\frac{1}{2} \frac{\mathrm{d}}{\mathrm{d} t} \varepsilon \eta\left\|\partial_{x} \omega\right\|^{2}$. The only dangerous term in this expression arises when we estimate $\left\langle\partial_{x}\left\{\omega_{\mathrm{s}}, \mathcal{L} \omega\right\}, \partial_{x} \omega\right\rangle$ by $\varepsilon \eta C\|\nabla \omega\|^{2}$. The latter term, however, can be absorbed into the left-hand side of (2.12) for $m=0$ provided we choose $\eta>0$ sufficiently small. We omit the details as they are straightforward. We remark, however, that we need to eliminate the aforementioned bad term, since we would otherwise only obtain the estimate $E_{m}(t) \leq C \int_{0}^{t} E_{m}(s) \mathrm{d} s+\varepsilon^{2}+\ldots$ which does not improve (1.8). We also note that we will not find better estimates by computing $\frac{1}{2} \frac{\mathrm{d}}{\mathrm{d} t} \mathcal{N}\left(\partial_{x} \tilde{\omega}\right)$ since we break the Hamiltonian structure by taking the $x$-derivative of (2.9) so that the useful identity (2.16) is no longer true. 
Theorem 2 If Hypothesis 2 is met, then there exist numbers $C>0, \nu \in(1 / 2,1)$ and $\kappa>0$ such that

$$
\|u(t)\|_{L^{\infty}}+\|\nabla u(t)\|_{L^{\infty}} \leq C \varepsilon^{\nu} \quad \forall t \in\left[0, \varepsilon^{-\kappa}\right)
$$

where $\varepsilon \in\left(0, \varepsilon_{0}\right]$.

The above theorem shows that the perturbed flow $u^{\varepsilon}(t, x, y)$ satisfies Hypothesis 1 whenever the stationary flow $u_{\mathrm{s}}(x, y)$ satisfies Hypothesis 2. In particular, the Melnikov calculations in Theorem 1 are applicable to these base flows.

Proof. For any fixed $\gamma$ with $3 / 4<\gamma<1$, we can find $\kappa$ and $\theta$ with $0<\kappa<(1-\gamma) / 3$ and $1 / 2<\theta \leq 1$ such that $\nu:=\gamma-\kappa-\theta / 2$ satisfies $1 / 2<\nu<1$. Proposition 2 then implies that

$$
\|u(t)\|_{L^{\infty}} \stackrel{(2.20)}{\leq} C\|\nabla \omega\|^{\theta}\|\omega\|^{1-\theta} \leq C \varepsilon^{\gamma(1-\theta)+(\gamma-1 / 2) \theta} \leq C \varepsilon^{\gamma-\theta / 2}=C \varepsilon^{\nu}
$$

uniformly in $t \in\left[0, \varepsilon^{-\kappa}\right)$. This gives the first part of (2.24).

To estimate $\|\nabla u\|_{L^{\infty}}$, we shall use the classical estimate [4]

$$
\|\nabla u(t)\|_{L^{\infty}} \leq C\left(1+\ln \|\omega(t)\|_{H^{2}}\right)\|\omega(t)\|_{L^{\infty}}
$$

Thus, we need to bound $\|\omega\|_{L^{\infty}}$. We remark that the estimate

$$
\|\omega(t)\|_{L^{\infty}} \stackrel{(2.20)}{\leq} C\|\Delta \omega\|^{\theta}\|\omega\|^{1-\theta} \leq C \varepsilon^{\gamma(1-\theta)+(\gamma-1) \theta} \leq C \varepsilon^{\gamma-\theta},
$$

which holds for $1 / 2<\theta \leq 1$, is not sufficient since we cannot achieve $\gamma-\theta>1 / 2$ since $\gamma<1$. Instead, we estimate $\|\omega(t)\|_{L^{\infty}}$ indirectly from (2.8)

$$
\partial_{t} \omega+\left(u_{\mathrm{s}}+u\right) \cdot \nabla \omega-\varepsilon \Delta \omega=\varepsilon F-u \cdot \nabla \omega_{\mathrm{s}}
$$

by using it to derive bounds for $\|\omega(t)\|_{L^{p}}$ for each $p \geq 2$. From (2.27), we obtain

$$
\frac{\mathrm{d}}{\mathrm{d} t}\|\omega(t)\|_{L^{p}}^{p}=p \int_{\mathbb{T}^{2}}\left[-\omega^{p-1}\left(u+u_{\mathrm{s}}\right) \cdot \nabla \omega+\varepsilon \omega^{p-1} \Delta \omega-\omega^{p-1} u \cdot \nabla \omega_{\mathrm{s}}+\varepsilon F \omega^{p-1}\right] \mathrm{d} x \mathrm{~d} y .
$$

We consider each of the terms on the right-hand side separately. First, since $\nabla \cdot\left(u+u_{\mathrm{s}}\right)=0$, we see that

$$
p \int_{\mathbb{T}^{2}}\left(u+u_{\mathrm{S}}\right) \cdot(\nabla \omega) \omega^{p-1}=\int_{\mathbb{T}^{2}}\left(u+u_{\mathrm{s}}\right) \cdot \nabla\left(\omega^{p}\right)=-\int_{\mathbb{T}^{2}} \omega^{p} \nabla \cdot\left(u+u_{\mathrm{S}}\right)=0 .
$$

Integration by parts also shows that

$$
p \varepsilon \int_{\mathbb{T}^{2}} \omega^{p-1} \Delta \omega=-p(p-1) \int_{\mathbb{T}^{2}} \omega^{p-2}|\nabla \omega|^{2} \leq 0
$$

since $p \geq 2$. For the remaining two terms, we use the Hölder inequality and obtain

$$
\begin{aligned}
p \int_{\mathbb{T}^{2}} \omega^{p-1} u \cdot \nabla \omega_{\mathrm{s}}+p \varepsilon \int_{\mathbb{T}^{2}} \omega^{p-1} F & \leq p\left\|\omega^{p-1}\right\|_{L^{q}}\left(\left\|u \cdot \nabla \omega_{\mathrm{s}}\right\|_{L^{p}}+\varepsilon\|F\|_{L^{p}}\right) \\
& \leq p C\left(4 \pi^{2}\right)^{1 / p}\|\omega\|_{L^{p}}^{p-1}\left(\varepsilon+\|u\|_{L^{\infty}}\right)
\end{aligned}
$$


where $C$ is independent of $p$, and $q$ is chosen according to $1 / q=1-1 / p$. Substituting these estimates into (2.28), we get

$$
p\|\omega(t)\|_{L^{p}}^{p-1} \frac{\mathrm{d}}{\mathrm{d} t}\|\omega(t)\|_{L^{p}}=\frac{\mathrm{d}}{\mathrm{d} t}\|\omega(t)\|_{L^{p}}^{p} \leq p C\left(4 \pi^{2}\right)^{1 / p}\|\omega\|_{L^{p}}^{p-1}\left(\varepsilon+\|u\|_{L^{\infty}}\right)
$$

and therefore

$$
\|\omega(t)\|_{L^{p}} \leq C\left(4 \pi^{2}\right)^{1 / p} \int_{0}^{t}\left(\varepsilon+\|u(s)\|_{L^{\infty}}\right) \mathrm{d} s
$$

for every $t \in\left[0, \varepsilon^{-\kappa}\right)$. Taking the limit $p \rightarrow \infty$ and using (2.25), we see that

$$
\|\omega(t)\|_{L^{\infty}} \leq C \varepsilon^{\gamma-\kappa-\theta / 2} .
$$

which, once substituted into (2.26), gives

$$
\|\nabla u(t)\|_{L^{\infty}} \leq C|\ln \varepsilon| \varepsilon^{\gamma-\kappa-\theta / 2} \quad \forall t \in\left[0, \varepsilon^{-\kappa}\right) .
$$

The choices we made above guarantee that $1 / 2<\gamma-\kappa-\theta / 2<1$. Thus, choosing $\nu$ so that $1 / 2<\nu<\gamma-\kappa-\theta / 2$ establishes the desired estimate (2.24).

\section{The two-dimensional viscous barotropic equations}

We consider the $\beta$-barotropic Euler equation

$$
\partial_{t} \Delta \psi^{0}+\left\{\psi^{0}, \Delta \psi^{0}\right\}+\beta \partial_{x} \psi^{0}=0
$$

and the associated barotropic Navier-Stokes equation

$$
\partial_{t} \Delta \psi^{\varepsilon}+\left\{\psi^{\varepsilon}, \Delta \psi^{\varepsilon}\right\}+\beta \partial_{x} \psi^{\varepsilon}=\varepsilon\left(\Delta^{2} \psi^{\varepsilon}+f\right)
$$

on $\mathbb{T}^{2}$ and present two classes of solutions that satisfy the long-term estimates required in Hypothesis 1 .

\subsection{Example 1 continued}

The stream functions

$$
\psi_{\mathbf{s}}(t, x, y)=A \sin (x-c t+a)+B \sin (y+b), \quad c=-\beta
$$

provide an interesting class of solutions to (3.1) that exhibit closed stream lines. Using the new independent variable $\xi=x-c t$, these solutions become steady states of

$$
\partial_{t} \Delta \psi+\{\psi, \Delta \psi\}+\beta \partial_{\xi}(\psi+\Delta \psi)=0
$$

where all differential operators act on the variables $(\xi, y)$. The associated potential vorticity

$$
q_{\mathrm{s}}(\xi, y)=\Delta \psi_{\mathrm{s}}(\xi, y)+\beta y
$$




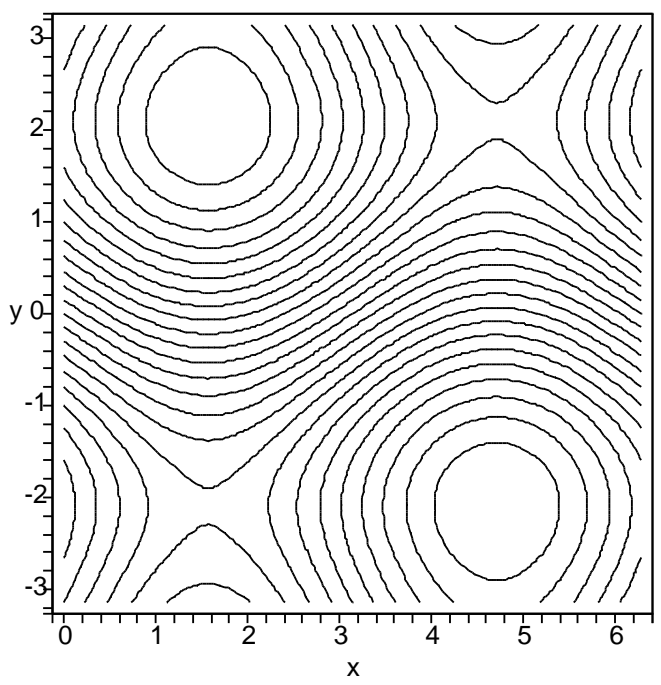

Figure 3: The level sets (streamlines) of the stream function $\psi_{\mathbf{s}}(x, y)=\sin x+\sin y+c y$ are plotted for $c=0.5$.

is time-dependent and conserved by the Lagrangian dynamics. For appropriate values of $A$ and $B$, the ODE (1.3) exhibits separatrices as illustrated in Figure 3 where the level sets of $q_{\mathrm{s}}(\xi, y)$ are plotted.

Thus, it is desirable to take $\psi_{\mathrm{s}}$ as an initial condition for (3.2). In the moving frame $\xi=x-c t$, (3.2) becomes

$$
\partial_{t} \Delta \psi^{\varepsilon}+\left\{\psi^{\varepsilon}, \Delta \psi^{\varepsilon}\right\}+\beta \partial_{\xi}\left(\psi^{\varepsilon}+\Delta \psi^{\varepsilon}\right)=\varepsilon\left(\Delta^{2} \psi^{\varepsilon}+f\right),
$$

and we need to establish long-time estimates of $\left\|\psi^{\varepsilon}(t)-\psi_{\mathrm{s}}\right\|$. Setting $\omega=\Delta\left(\psi^{\varepsilon}-\psi_{\mathrm{s}}\right)$ and $\omega_{\mathrm{s}}=\Delta \psi_{\mathrm{s}}$, we see that $\omega$ satisfies the equation

$$
\partial_{t} \omega=\left\{\omega_{\mathrm{s}}, \mathcal{L} \omega\right\}-\beta \partial_{\xi}(\mathcal{L} \omega)-J \nabla \Delta^{-1} \omega \cdot \nabla \omega+\varepsilon(\Delta \omega+F)
$$

with initial condition

$$
\omega(0, \xi, y)=0
$$

where $F=\nabla \wedge f+\Delta \omega_{\mathrm{s}}$ and $\mathcal{L}=\mathbb{1}+\Delta^{-1}$. The following proposition shows that the long-time behavior of (3.4) is similar to that of the Euler equation.

Proposition 3 The velocity field associated with the solution of (3.4)-(3.5) satisfies (2.24).

Proof. First, note that $\mathcal{L}=\mathbb{1}+\Delta^{-1}$ satisfies Hypothesis 2 on account of the computations in Example 1. Using the bilinear form

$$
\mathcal{N}(\omega)=\int_{\mathbb{T}^{2}} \omega \mathcal{L} \omega
$$

we claim that the conclusion of Proposition 2 is also true for (3.4). Indeed, the key observation is that

$$
\beta\left\langle\partial_{\xi}(\mathcal{L} \omega), \mathcal{L} \omega\right\rangle=0
$$




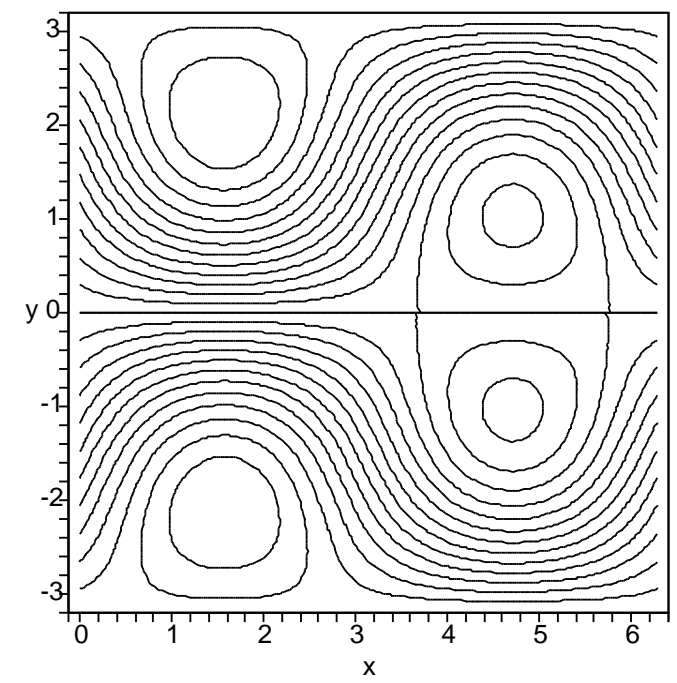

Figure 4: The level sets (streamlines) of the stream function $\psi_{\mathrm{s}}(x, y)=\sin x \sin y+c y$ associated with Rossby waves are plotted for $c=0.5$.

which means that the proof of Proposition 2 remains valid. Thus, it remains to establish the same $L^{\infty}$-bounds as in Theorem 2. Following the proof of Theorem 2, we need to estimate the additional term $p \int_{\mathbb{T}^{2}} \omega^{p-1} \partial_{\xi}(\mathcal{L} \omega)$. Using $\mathcal{L}=\mathbb{1}+\Delta^{-1}$, we get

$$
p \int_{\mathbb{T}^{2}} \omega^{p-1} \partial_{\xi}(\mathcal{L} \omega)=\int_{\mathbb{T}^{2}}\left(\partial_{\xi}\left(\omega^{p}\right)+p \omega^{p-1} \partial_{\xi} \Delta^{-1} \omega\right)=\int_{\mathbb{T}^{2}} p \omega^{p-1} \partial_{\xi} \Delta^{-1} \omega
$$

and Hölder's inequality gives

$$
\left|\int_{\mathbb{T}^{2}} p \omega^{p-1} \partial_{\xi} \Delta^{-1} \omega\right| \leq p\left(4 \pi^{2}\right)^{1 / p}\left\|\partial_{\xi} \Delta^{-1} \omega\right\|_{L^{\infty}}\|\omega\|_{L^{p}}^{p-1} \leq p C\left(4 \pi^{2}\right)^{1 / p}\|u\|_{L^{\infty}}\|\omega\|_{L^{p}}^{p-1}
$$

Thus, this term gives the same estimate as (2.29), and the remaining part of the proof is identical to the proof of Theorem 2 .

\subsection{Rossby waves}

Next, we consider the Rossby waves whose stream function is given by

$$
\psi_{\mathrm{s}}(t, x, y)=A \sin (x-c t) \sin (y), \quad c=-\frac{\beta}{2} .
$$

The separatrices for the Lagrangian dynamics in the moving frame are plotted in Figure 4.

We denote by $\psi^{\varepsilon}$ the solution of (3.3) with initial condition $\psi_{\mathrm{s}}$ and set again $\omega=\Delta\left(\psi^{\varepsilon}-\psi_{\mathrm{s}}\right)$, which then satisfies (3.4), where $\mathcal{L}$ is given by

$$
\mathcal{L}=\frac{1}{2} \mathbb{1}+\Delta^{-1}
$$

Thus, the theory developed so far does not directly apply since $\mathcal{L}$ has negative eigenvalues. Nevertheless, Hypothesis 1 is met provided the forcing term $f$ satisfies certain conditions. 
Proposition 4 If the function $f(t,(x+y) / 2,(x-y) / 2)$ is $2 \pi$-periodic in $(x, y)$, then the velocity field associated with the Rossby-wave solution of (3.4)-(3.5) satisfies (2.24).

The assumption on the forcing term is true, for instance, if $f(t, x, y)$ is $\pi$-periodic in $(x, y)$.

Proof. We introduce the new coordinates $(\check{x}, \check{y})$ via

$$
(\check{x}, \check{y})=(x+y, x-y), \quad(x, y)=\left(\frac{\check{x}+\check{y}}{2}, \frac{\check{x}-\check{y}}{2}\right) .
$$

The transformed stream function

$$
\check{\psi}^{\varepsilon}(t, \check{x}, \check{y}):=\psi^{\varepsilon}(t, x, y)
$$

then satisfies the PDE

$$
\partial_{t} \Delta \check{\psi}^{\varepsilon}=2\left\{\check{\psi}^{\varepsilon}, \Delta \check{\psi}^{\varepsilon}\right\}+2 \varepsilon \Delta^{2} \check{\psi}^{\varepsilon}-\frac{\beta}{2}\left(\partial_{\check{x}} \check{\psi}^{\varepsilon}+\partial_{\check{y}} \check{\psi}^{\varepsilon}\right)+\frac{\varepsilon}{2} \check{f}
$$

on $\mathbb{T}^{2}$, where $\check{f}(t, \check{x}, \check{y})=f(t,(\check{x}+\check{y}) / 2,(\check{x}-\check{y}) / 2)$, and where the differential operators act on the new variables $(\check{x}, \check{y})$. Since the transformed base flow

$$
\check{\psi}_{\mathrm{s}}(t, \check{x}, \check{y})=\psi_{\mathrm{s}}\left(t, \frac{\check{x}+\check{y}}{2}-c t, \frac{\check{x}-\check{y}}{2}\right)=A \sin \left(\frac{\check{x}+\check{y}}{2}-c t\right) \sin \left(\frac{\check{x}-\check{y}}{2}\right)
$$

and the transformed forcing $\check{F}$ are both $2 \pi$-periodic in $(\check{x}, \check{y})$, so is the solution $\check{\psi}^{\varepsilon}$ of $(3.6)$.

Next, we introduce the moving frame coordinates $\xi=\check{x}-c t$ and $\eta=\check{y}-c t$ where $c=-\beta / 2$. Writing the vorticity $\check{\omega}=\Delta\left(\check{\psi}^{\varepsilon}-\check{\psi}_{\text {s }}\right)$ as a function of the independent variables $(t, \xi, \eta)$, we find that it satisfies the equation

$$
\partial_{t} \check{\omega}+\frac{\beta}{2}\left(\partial_{\xi} \check{\mathcal{L}} \check{\omega}+\partial_{\eta} \check{\mathcal{L}} \check{\omega}\right)=2\left\{\check{\omega}_{\mathrm{s}}, \check{\mathcal{L}} \check{\omega}\right\}+2 \varepsilon \Delta \check{\omega}+\varepsilon F-2 J \nabla \Delta^{-1} \check{\omega} \cdot \nabla \check{\omega}
$$

where the transformed operator $\check{\mathcal{L}}$ is now given by

$$
\check{\mathcal{L}}=\mathbb{1}+\Delta^{-1} .
$$

Thus, $\check{\mathcal{L}}$ satisfies Hypothesis 2, and Proposition 3 remains true since the additional term $\partial_{\eta} \check{\mathcal{L}} \check{\omega}$ can be treated in the same fashion as the term $\partial_{\xi} \check{\mathcal{L}} \check{\omega}$ that appeared in the proof of Proposition 3.

\section{Extensions to modified Navier-Stokes equations}

In Section 2, we incorporated viscosity by adding $\Delta u$, corresponding to molecular viscosity, to the Euler equation, yielding the classical Navier-Stokes equation. A major source of viscosity in problems from meteorology and oceanography is the effect of small-scale eddies on larger structures in the fluid flow. Smagorinsky [17] proposed a nonlinear viscosity force which yields the modified Navier-Stokes equation

$$
\partial_{t} u+(u \cdot \nabla) u+\nabla p=\varepsilon[\operatorname{div}(|S| S)+f], \quad \nabla \cdot u=0
$$


where $S=S(u)$ is the symmetric part of the velocity gradient given by

$$
S=S(u):=\frac{1}{2}\left(\nabla u+(\nabla u)^{*}\right) .
$$

Given the widespread use of (4.1) and other related models (see below), it is of interest to see whether the long-time estimate (5.1) can also be established for (4.1) as this would allow us to make prediction on the transport based on the Melnikov theory in Theorem 1.

Unfortunately, the Smagorinsky model seems difficult to attack. The main problem is to obtain sufficient regularity for solutions of (4.1). Indeed, for the Navier-Stokes equation, we have used the classical local existence theorem of smooth solutions to reduce the verification of Hypothesis 1 to establishing stability. Because of the singularity in the viscosity tensor at points where $S(u)$ vanishes, the Smagorinsky model does not fit into this framework: as far as we are aware, there is no local existence result for smooth solutions of (4.1).

Surprisingly, (4.1) has much better mathematical properties with regard to weak solutions than the classical Navier-Stokes equation: existence and uniqueness results for weak solutions have been proved in [10], even in 3d; we refer to [7] for further references.

To take into account some of the effects of the Smagorinsky model, we consider the nonlinear viscosity model

$$
\partial_{t} u+(u \cdot \nabla) u+\nabla p=\varepsilon[\operatorname{div}(\mathcal{T}(S))+f], \quad \nabla \cdot u=0
$$

with a stress tensor $\mathcal{T}(S)$ of the form

$$
\mathcal{T}(S)=b(S) S
$$

where $b: \mathbb{R}^{2 \times 2} \rightarrow \mathbb{R}$ is smooth and bounded away from zero so that

$$
b \in C^{\infty}\left(\mathbb{R}^{2 \times 2}, \mathbb{R}\right), \quad b(p) \geq b_{0}>0 \quad \forall p \in \mathbb{R}^{2 \times 2} .
$$

The example $b(S)=\left(1+|S|^{2}\right)^{\frac{1}{2}}$ is of particular interest as it incorporates both molecular and Smagorinsky viscosity. Note that the term $b(S)=1+|S|$ obtained by simply adding molecular and Smagorinsky viscosity is bounded away from zero but not smooth at $S=0$.

For stress tensors $\mathcal{T}(S)=b(S) S$ that obey (4.3), Theorem 2 can be readily extended:

Theorem 3 Assume that b satisfies (4.3). If Hypothesis 2 is met, then there exist numbers $C>0$, $\nu \in(1 / 2,1)$ and $\kappa>0$ such that the solution $u^{\varepsilon}$ of (4.2) with initial value $u_{\mathrm{s}}$ enjoys the estimate (2.24).

Proof. The strategy is the same as for the proof of Theorem 2. As before, we need to establish the estimates stated in Proposition 2. Setting $u=u^{\varepsilon}-u_{\mathrm{s}}$ and $\omega=\operatorname{curl} u$, we see that $\omega$ satisfies the equation

$$
\partial_{t} \omega+u_{\mathrm{s}} \cdot \nabla \omega+u \cdot \nabla \omega_{\mathrm{s}}+u \cdot \nabla \omega=\varepsilon\left[b\left(S\left(u+u_{\mathrm{s}}\right)\right) \Delta \omega+F\right]+Q,
$$

where

$$
Q=\varepsilon \operatorname{curl} \nabla \cdot\left[b\left(S\left(u+u_{\mathrm{s}}\right)\right) S\left(u_{\mathrm{s}}\right)\right]+\varepsilon \operatorname{curl}\left[S(u) \nabla\left(b\left(S\left(u+u_{\mathrm{s}}\right)\right)\right)\right] .
$$


Equation (4.4) is a quasilinear parabolic equation for which we have local existence of solutions in $\stackrel{\circ}{H}^{s}$ for $s>4$ (see, for instance, [18, Chapter 15]). Thus, we can define $T_{\varepsilon}$ as in the proof of Proposition 2 and establish the estimate (2.12) by the same methods. We claim that the new nonlinear terms can be treated as before by using Gagliardo-Nirenberg-Moser estimates (see again [18]): The key point is that these terms make only harmless contributions to the estimates because all new higher-order derivatives which appear have a factor $\varepsilon$ in front of them. To illustrate this claim, we explain in detail how the term

$$
\sum_{|\alpha|=m} I_{\alpha}:=\sum_{|\alpha|=m} \varepsilon \int_{\mathbb{T}^{2}} \varepsilon^{|\alpha|} \partial^{\alpha}\left(b\left(S\left(u+u_{\mathrm{s}}\right)\right) \Delta \omega\right) \partial^{\alpha} \omega \mathrm{d} x \mathrm{~d} y
$$

is treated. First, thanks to the a priori assumption (2.11) and the Gagliardo-Nirenberg inequalities (2.20), we have

$$
\|\nabla u\|_{L^{\infty}} \leq \varepsilon^{\gamma-\frac{3}{4}}, \quad\left\|\nabla^{2} u\right\|_{L^{\infty}} \leq \varepsilon^{\gamma-\frac{5}{4}}, \quad\|\Delta \omega\|_{L^{\infty}} \leq \varepsilon^{\gamma-\frac{7}{4}}
$$

for $t<T_{\varepsilon}$. To estimate $I_{\alpha}$, we write

$$
\mathrm{I}_{\alpha}=\varepsilon \int_{\mathbb{T}^{2}} \varepsilon^{|\alpha|} b \Delta\left(\partial^{\alpha} \omega\right) \partial^{\alpha} \omega+\varepsilon \int_{\mathbb{T}^{2}} \varepsilon^{|\alpha|}\left[\partial^{\alpha}, b\right](\Delta \omega) \partial^{\alpha} \omega=: \mathrm{II}_{\alpha}+\mathrm{III}_{\alpha}
$$

Upon integrating by parts and using assumption (4.3), the estimate (4.6) and Young's inequality, we get

$$
\begin{aligned}
\mathrm{II}_{\alpha} & \leq-\varepsilon \varepsilon^{|\alpha|} b_{0}\left\|\nabla \partial^{\alpha} \omega\right\|^{2}+C \varepsilon \varepsilon^{|\alpha|} \int_{\mathbb{T}^{2}}\left|\nabla \partial^{\alpha} \omega\right|\left|\partial^{\alpha} \omega\right|\left(1+\left|\nabla^{2} u\right|\right) \\
& \leq-\varepsilon \varepsilon^{|\alpha|} b_{0}\left\|\nabla \partial^{\alpha} \omega\right\|^{2}+C \varepsilon^{\gamma-\frac{1}{4}} \varepsilon^{|\alpha|}\left\|\nabla \partial^{\alpha} \omega\right\|\left\|\partial^{\alpha} \omega\right\| \\
& \leq-\varepsilon \varepsilon^{|\alpha|} \frac{b_{0}}{2}\left\|\nabla \partial^{\alpha} \omega\right\|^{2}+C \varepsilon^{2 \gamma-\frac{3}{2}} \varepsilon^{|\alpha|}\left\|\partial^{\alpha} \omega\right\|^{2} .
\end{aligned}
$$

To estimate III $_{\alpha}$, we write

$$
\mathrm{III}_{\alpha} \leq C \varepsilon \varepsilon^{|\alpha|}\left\|\partial^{\alpha} \omega\right\|\left\|\left[\partial^{\alpha}, b\right] \Delta \omega\right\|,
$$

and use commutator and Gagliardo-Nirenberg-Moser estimates (see [18, Chapter 13]) to get

$$
\begin{aligned}
\left\|\left[\partial^{\alpha}, b\right] \Delta \omega\right\| & \leq C\left(\|b\|_{H^{|\alpha|}}\|\Delta \omega\|_{L^{\infty}}+\|\nabla b\|_{L^{\infty}}\|\Delta \omega\|_{H^{|\alpha|-1}}\right) \\
& \leq C\left(\left(1+\|\nabla u\|_{H^{|\alpha|}}\right)\|\Delta \omega\|_{L^{\infty}}+\left(1+\left\|\nabla^{2} u\right\|_{L^{\infty}}\right)\|\nabla \omega\|_{H^{|\alpha|}}\right) .
\end{aligned}
$$

Finally, we can combine (4.7), (4.8) and (4.9) to get, upon using (4.6) and Young's inequality,

$$
\sum_{|\alpha|=m} \mathrm{I}_{\alpha} \leq-\varepsilon \frac{b_{0}}{4} \sum_{|\alpha|=m} \varepsilon^{|\alpha|}\left\|\nabla \partial^{\alpha} \omega\right\|^{2}+C\left(\varepsilon^{2 \gamma-\frac{3}{2}}+\varepsilon^{\gamma-\frac{3}{4}}\right) E_{m} .
$$

Lastly, we note that the second term in the above estimate is small for $\gamma>3 / 4$, which finishes the analysis for the term (4.5). We omit the analysis of the other terms which is quite analogous to the one given above. 


\section{Discussion}

Our motivation for this paper has been to determine the nature of transport that occurs when a small amount of viscosity and forcing is added to an inviscid stationary fluid flow. Thus, starting with a smooth stationary fluid flow $u_{\mathrm{s}}(x, y)$ of the Euler equation $(1.6)$ on $\mathbb{T}^{2}$, we considered the solution $u^{\varepsilon}(t, x, y)$ of the Navier-Stokes equation (1.7) with initial condition $u^{\varepsilon}(0, x, y)=u_{\mathrm{s}}(x, y)$, where $\varepsilon>0$ is the coefficient of viscosity. We showed in earlier work [3, 15] that Melnikov theory can be used to make predictions about transport for $0<\varepsilon \ll 1$ over time scales of the order $\varepsilon^{-\kappa}$ for $\kappa>0$ provided there are numbers $C>0$ and $\nu \in\left(\frac{1}{2}, 1\right]$ such that

$$
\left\|u^{\varepsilon}(t)-u_{\mathrm{s}}\right\|_{W^{1, \infty}\left(\mathbb{T}^{2}\right)} \leq C \varepsilon^{\nu}
$$

for $0 \leq t \leq \varepsilon^{-\kappa}$. In this paper, we established (5.1) in Theorem 2 whenever the underlying stationary flow $u_{\mathrm{s}}(x, y)$ satisfies Hypothesis 2 on the spectrum of a certain operator $\mathcal{L}$ whose coefficients depend on the profile $u_{\mathrm{s}}(x, y)$. We gave several example flows, including those induced by the stream functions

$$
\psi_{\mathrm{s}}(x, y)=A \sin (x+a)+B \sin (y+b)
$$

for which Hypothesis 2, and therefore the estimate (5.1), is true.

We also considered solutions of the $\beta$-barotropic Navier-Stokes equations (3.2) starting with a solution to the $\beta$-barotropic Euler equation (3.1). We proved that the estimate (5.1) holds for the Rossby waves with stream function

$$
\psi_{\mathbf{s}}(t, x, y)=A \sin \left(x+\frac{\beta t}{2}\right) \sin (y)
$$

and for the fluid flows associated with the stream function

$$
\psi_{\mathrm{s}}(t, x, y)=A \sin (x+\beta t)+B \sin (y) .
$$

In Section 4, we established the estimate (5.1) for the modified Navier-Stokes equation

$$
\partial_{t} u+(u \cdot \nabla) u+\nabla p=\varepsilon[\operatorname{div}(\mathcal{T}(S))+f], \quad \nabla \cdot u=0
$$

when the stress tensor $\mathcal{T}(S)$ is of the form $\mathcal{T}(S)=b(S) S$ with

$$
b \in C^{\infty}\left(\mathbb{R}^{2 \times 2}, \mathbb{R}\right), \quad b \geq b_{0}>0,
$$

where $S=S(u)$ denotes the symmetric part of $\nabla u$. The restriction to smooth and positive functions $b$ excludes the Smagorinsky model [17] for which $b(S)=|S|$. The main obstacle for extending our results to functions such as $b(S)=|S|$ is the lack of regularity theory for solutions of (5.2) when (5.3) is not met.

Lastly, we mention that we focused entirely on periodic boundary conditions. It would be interesting to see to which extent our results, and specifically the estimate (5.1), can be generalized to other boundary conditions. 
Acknowledgments C.K.R.T. Jones was partially supported by the National Science Foundation through grant DMS-0410267 and by the Office of Naval Research through grant N00014-92-J-1481. B. Sandstede was partially supported by the Alfred P Sloan Foundation and by the National Science Foundation through grant DMS-0203854.

\section{References}

[1] D.G. Andrews. On the existence of nonzonal flows satisfying sufficient conditions for stability. Geophys. Astrophys. Fluid Dynamics 28 (1984) 243-256.

[2] V.I. Arnold. An a priori estimate in the theory of hydrodynamic stability. Izv. Vyssh. Uchebn. Zaved. Matematika 54 (1966) 3-5. [English transl.: Amer. Math. Soc. Transl. 79 (1969) 267-69].

[3] H.S. Balasuriya, C.K.R.T. Jones and B. Sandstede. Viscous perturbations of vorticity conserving flows and separatrix splitting. Nonlinearity 11 (1998) 47-77.

[4] J.T. Beale, T. Kato and A. Majda. Remarks on the breakdown of smooth solutions for the 3-D Euler equations. Comm. Math. Phys. 1 (1984) 61-66.

[5] M.G. Brown and R.M. Samelson. Particle motion in vorticity-conserving, two-dimensional incompressible flows. Phys. Fluids 6 (1994) 2875-2876.

[6] R. Fjortoft. Application of integral theorems in deriving criteria of stability for laminar flows and for the baroclinic circular vortex. Geofys. Pub. Oslo 17 (1950) 1-52.

[7] S. Friedlander and N. Pavlović. Remarks concerning modified Navier-Stokes equations. Discr. Cont. Dynam. Syst. 10 (2004) 269-288.

[8] S. Friedlander, W. Strauss and M. Vishik. Nonlinear instability in an ideal fluid. Ann. Inst. H. Poincaré Anal. Non Linéaire 14 (1997) 187-200.

[9] D.D. Holm, J.E. Marsden, T. Ratiu and A. Weinstein. Nonlinear stability of fluid and plasma equilibria. Phys. Rep. 123 (1985) 1-116.

[10] O.A. Ladyzhenskaya. The Mathematical Theory of Viscous Incompressible Flow. Gordon and Breach, New York, 1969.

[11] C. Marchioro and M. Pulvirenti. Mathematical Theory of Incompressible Nonviscous Fluids. SpringerVerlag, New York, 1994.

[12] J.M. Ottino. Mixing, chaotic advection, and turbulence. Ann. Rev. Fluid Mech. 22 (1990) 207-253.

[13] Lord Rayleigh. On the stability, or instability, of certain fluid motions. Proc. London Math. Soc. 11 (1880) $57-70$.

[14] R.M. Samelson. Fluid exchange across a meandering jet. J. Phys. Oceanography 22 (1992) 431-440.

[15] B. Sandstede, S. Balasuriya, C.K.R.T. Jones and P. Miller. Melnikov theory for finite time vector fields. Nonlinearity 13 (2000) 1357-1377.

[16] R. Shvidkoy and Y. Latushkin. The essential spectrum of the linearized 2D Euler operator is a vertical band. Contemp. Math. 327 (2003) 299-304. 
[17] J. Smagorinsky. General circulation experiments with the primitive equations. Monthly Weather Rev. 91 (1963) 99-164.

[18] M.E. Taylor. Partial Differential Equations III. Springer-Verlag, New York, 1997.

[19] R. Temam. Navier-Stokes Equations and Nonlinear Functional Analysis. CBMS-NSF Regional Conference Series in Applied Mathematics. SIAM, Philadelphia, 1983.

[20] M. Vishik. Spectrum of small oscillations of an ideal fluid and Lyapunov exponents. J. Math. Pures Appl. 75 (1996) 531-558. 